\title{
Desafios e recomendações para o cuidado intensivo de adultos críticos com doença de coronavírus 2019 (COVID-19)
}

Challenges and recommendatios for the intensive care of adults with coronavirus disease 2019 (COVID-19)

Resumo: A pandemia pela doença de coronavírus 2019 (COVID-19) representa enorme desafio para o sistema de saúde em todo o mundo. Foi nosso objetivo revisar e sintetizar os principais desafios associados ao cuidado intensivo do paciente adulto crítico com COVID-19 e apresentar evidências de recomendações. Trata-se de revisão integrativa de literatura, baseada na experiência de outros países, que tem o potencial de instrumentalizar os profissionais de saúde intensivistas para a prática assistencial ao paciente crítico com COVID-19. Dentre os principais desafios a serem enfrentados pelas equipes de unidades de terapia intensiva (UTI), evidenciados pela literatura, sintetizamos nesse trabalho: a manifestação clínica e diagnóstico para isolamentos rápidos; o manejo da insuficiência respiratória aguda; o papel das terapias medicamentosas reaproveitadas, experimentais e adjuvantes; a prevenção de infecções para pacientes e profissionais; a gestão da mortalidade de pacientes e o gerenciamento da equipe de UTI. O estado atual de conhecimento sobre a COVID-19 é considerado insuficiente e cheio de lacunas, o que configura outro desafio e enseja a necessidade de realização de mais pesquisas clínicas.

Palavras-Chave: COVID-19; Unidade de Terapia Intensiva (UTI); Desafios e Recomendações.

Abstract: A pandemic for coronavirus disease 2019 (COVID-19) represents a huge challenge for the healthcare system worldwide. Our objective was to review and summarize the main challenges associated with the intensive care of adult critical patients with COVID-19 and presented the use of recommendations. It is an integrative literature review, experience from other countries, which has the potential to equip intensive care professionals in Brazil for a care practice for critical patients with COVID-19. Among the main challenges faced by the teams of intensive care units (ICU), evidenced by the literature, synthesized in this work: a clinical manifestation and diagnosis for rapid isolations; the management of acute respiratory failure; the role of reused drug therapies, experiments and adjuvants; the prevention of infections by patients and professionals; managing patient mortality and the management of the ICU team. The current state of knowledge about COVID-19 is considered insufficient and full of gaps, or represents another challenge and leads to the need to conduct different types of clinical research.

Key Words: COVID-19; Intensive Care Unit (ICU); Challenges and Recommendations. 


\section{INTRODUÇÃO}

Pacientes diagnosticados com a doença de coronavírus (CoV) 2019 (COVID-19) podem evoluir para Síndrome do Acometimento Respiratório Severo (SARS-CoV), com complicações por choque e falência de múltiplos órgãos ${ }^{1}$. Esses pacientes necessitam de cuidados intensivos ofertados na Unidade de Terapia Intensiva (UTI), que oferece Ventilação Mecânica (VM), Terapia de Substituição Renal (TSR) e, em casos mais graves, a Oxigenação por Membrana Extracorpórea (ECMO) ${ }^{1,2}$.

É nosso objetivo sintetizar os principais desafios associados ao cuidado intensivo do paciente adulto crítico com COVID-19 e apresentar evidências de recomendações. Trata-se de revisão integrativa de literatura, baseada na experiência de outros países, que tem o potencial de instrumentalizar os profissionais de saúde intensivistas para a prática assistencial ao paciente crítico com COVID-19.

COVID-19 é o nome dado a doença causada pelo SARS- CoV-2, terceira infecção pelo vírus da família coronaviridae, em duas décadas ${ }^{2}$. A disseminação da doença é rápida e em grande escala, o que gerou o estado de pandemia em $2020^{3,4}$. Os dados epidemiológicos da doença alteram-se diariamente. Registramos os dados de 16 de abril de 2020: 2.090.110 pessoas foram diagnosticadas com COVID-19 no mundo ${ }^{5}$, com 139.469 mortes; o Brasil apresentava 30.420 confirmações, 1.924 óbitos e taxa de letalidade de 6\%; o número de hospitalizados ultrapassava 9 mil no Brasil ${ }^{12 .}$ e o Distrito Federal apresentava 726 casos confirmados e 20 óbitos, com letalidade de $2,8 \%$.

Estudo da OMS-China afirma que nem todos os casos críticos de COVID-19 foram admitidos na UTI, pois as admissões dependem da gravidade da doença e da capacidade da UTI do sistema de saúde ${ }^{4}$. O Brasil conta com 40,6 mil leitos de UTI, 23 mil pertencentes ao Sistema Único de Saúde (SUS), destes, 14.869 são destinados a adultos ${ }^{6}$. Esse número de leitos de UTI pode não ser suficiente para a assistência aos pacientes críticos com COVID-19, concomitantemente a pacientes de outras clínicas.

Desse modo, profissionais de UTI, administradores de hospitais, governos e formuladores de políticas públicas devem se preparar rapidamente para um aumento substancial 
na capacidade de cuidados intensivos, ou vamos correr o risco de sermos sobrecarregados pela pandemia.

\section{MÉTODOS}

Realizou-se revisão integrativa de literatura, buscando a experiência adquirida por outros serviços de UTI, em diferentes países, no enfrentamento ao COVID-19. Consideramos artigos indexados publicados de janeiro a abril de 2020, nas bases de dados National Library of Medicine (PubMed), Scientific Electronic Library Online (SciELO) e Literatura LatinoAmericana e do Caribe em Ciências da Saúde (LILACS), utilizando os idiomas inglês e português, os descritores, correlacionados foram: UTI/ICU; terapia intensiva/intensive therapy; paciente crítico/critical patient e COVID-19/coronavírus. A pergunta norteadora da revisão foi: quais são os principais desafios relatados e as principais recomendações para os profissionais de saúde intensivistas por parte dos países que estão lidando com a assistência do paciente crítico adulto com COVID-19? Incluímos os artigos disponíveis na íntegra em língua inglesa e portuguesa sobre a temática publicados no ano de 2020 e acessados em abril de 2020. Foram excluídos trabalhos que não versavam sobre a temática eleita. Incluímos um total de 57 publicações. Cumprimos as seguintes etapas: elaboração da pergunta norteadora; eleição de critérios de inclusão e exclusão; definição dos descritores, busca nas bases de dados; análise crítica dos estudos e discussão dos resultados e apresentação da síntese do conhecimento produzido.

\section{RESULTADOS E DISCUSSÃO}

A quase totalidade das referências utilizadas são de publicações na língua inglesa. Considerando o propósito do nosso estudo, organizamos a síntese dos resultados em temas relacionados aos principais desafios elencados pelos autores, com as respectivas recomendações. Consideramos, ainda, em cada tema tratado, a discussão dos achados.

\section{Características clínicas e Diagnóstico}

Os sintomas da COVID-19 são inespecíficos e sua apresentação pode variar de formas assintomáticas até pneumonia grave e óbito. Em uma revisão da WHO-China que inclui 55924 pacientes confirmados, $80 \%$ destes apresentaram doença leve ou moderada, $13,8 \%$ doença severa (dispneia, frequência respiratória $\geq 30 /$ minuto, saturação de oxigênio $\leq 93 \%, \mathrm{~Pa} 02 / \mathrm{FiO} 2$ ratio $<300$ e/ou infiltrado pulmonar $>50 \%$ do tecido pulmonar em $24 / 48$ horas) e $6,1 \%$ foram 
críticos (insuficiência respiratória, choque séptico e/ou falência múltipla de órgãos). Indivíduos com maior risco para doença severa e óbito são aqueles com mais de 60 anos e com comorbidades, como hipertensão arterial, diabetes mellitus, doença cardiovascular, doença pulmonar crônica e câncer ${ }^{4}$.

Os sintomas mais comuns de pacientes críticos são febre, tosse, dispneia e fadiga. Sintomas menos comuns são cefaleia, tontura, dor abdominal, diarreia, náusea e vômito $^{3,7}$.

A maioria dos pacientes críticos apresenta opacidades bilaterais na tomografia computadorizada (TC) e radiografia de tórax, sendo comum padrão em vidro fosco e consolidação ${ }^{7,8,9}$. Linfopenia é um dado laboratorial comum em pacientes críticos, sendo que os óbitos apresentaram linfopenia mais severa ${ }^{3}$. Em internados com COVID-19, as alterações laboratoriais mais comuns são linfopenia, aumento do tempo da protrombina e aumento da desidrogenase láctica ${ }^{7}$.

A principal complicação é a síndrome de angústia respiratória aguda (SDRA). Outras complicações incluem injúria renal aguda, disfunção hepática, injúria cardíaca, choque e infecção secundária ${ }^{3,7}$.

A Organização Mundial da Saúde (OMS) declarou pandemia da COVID-19 e, como consequência, qualquer paciente com evidência de infecção respiratória deve ser considerado potencialmente infectado pelo SARS-CoV-2. O diagnóstico é baseado no teste de RT-PCR de amostras de swab naso e orofaríngeo e de vias aéreas inferiores. Um único resultado negativo do RT-PCR não exclui o diagnóstico, podendo ser repetido em outra amostra do trato respiratório quando houver discordância com o quadro clínico epidemiológico. Por ter elevada especificidade, um único resultado positivo de RT-PCR é suficiente para iniciar tratamento e protocolo de precauções ${ }^{10}$.

O lavado broncoalveolar deve ser realizado somente se houver indicação e com as precauções adequadas devido ao risco de aerossolização e exposição dos profissionais de saúde. Pelo mesmo motivo, a indução de escarro também deve ser evitada. Amostras de aspirado traqueal parecem carregar menor risco de formação de aerossol e podem ser obtidas sem a necessidade de desconectar o paciente do ventilador. Amostras das vias aéreas inferiores apresentam maior potencial diagnóstico do que as das vias aéreas superiores em pacientes com pneumonia, e devem ser obtidas sempre que possível ${ }^{10 .}$ 
Em estudo, amostras de lavado broncoalveolar mostraram as maiores taxas de positividade (93\%), seguido de escarro (72\%), swab nasal (63\%), biópsia via fibrobroncoscopia (46\%), swab faríngeo (32\%), fezes (29\%), sangue (1\%) e urina (0\%). A testagem de amostras de diferentes sítios pode aumentar a sensibilidade e reduzir resultados falso-negativos ${ }^{11}$.

Os testes sorológicos visam detectar anticorpos IgG e IgM específicos produzido pelo corpo humano contra o SARS-CoV-2 ${ }^{12}$. Estudos recentes sugerem que a maioria dos pacientes realiza soroconversão entre 7 e 11 dias após exposição ao vírus. ${ }^{9}$ Além disso, uma vez que o desenvolvimento de uma resposta de anticorpo à infecção pode ser dependente do hospedeiro, os testes sorológicos com identificação de anticorpos IgG e IgM ao SARS-CoV-2 não são recomendados para a confirmação diagnóstica de pacientes com sintomas de início recente, embora apresentem boa acurácia e sensibilidade ${ }^{12,14}$.

A TC de tórax não deve ser usada isoladamente para diagnóstico ou rastreamento de COVID-19. Para se definir o diagnóstico, é preciso unir informações clínico-epidemiológicas aos exames RT-PCR e/ou sorologia. Os achados da TC para pacientes com suspeita de COVID19 não parecem influenciar desfechos. A TC pode ser realizada em pacientes com piora dos sintomas e/ou desenvolvimento de complicações ${ }^{15}$. A síntese das recomendações e desafios está no Quadro 1.

Quadro 1. Desafios e recomendações para o diagnóstico da COVID-19

\begin{tabular}{|l|l|}
\hline \multicolumn{1}{|c|}{ Desafios } & \multicolumn{1}{|c|}{ Recomendações } \\
\hline Quadro clínico de COVID-19 inespecífico & $\begin{array}{l}|c| \\
\text { COVID-19 }\end{array}$ \\
\hline Sensibilidade do RT-PCR desconhecida & - Realização de testes que atestem a \\
& $\begin{array}{l}\text { sensibilidade do RT-PCR } \\
- \text { Preferência para coleta de amostras das vias } \\
\text { respiratórias inferiores sempre que possível }\end{array}$ \\
\hline Não disponibilidade do RT-PCR em muitos & - Manter alto índice de suspeita de COVID- \\
serviços ou tempo elevado para resultados & 19 \\
& - Realizar TC de tórax se disponível \\
\hline Soroconversão tardia & - Diagnóstico sorológico não é recomendado \\
& em fase inicial do quadro clínico \\
\hline
\end{tabular}




\section{Manejo da insuficiência respiratória aguda}

A SDRA é uma forma de insuficiência respiratória caracterizada por uma inflamação da membrana alvéolo-capilar que gera aumento da permeabilidade e consequente alteração da ventilação e oxigenação pulmonar ${ }^{16}$. Constitui uma das principais complicações associada aos pacientes graves com COVID-19, que se instala rapidamente e pode ser fatal ${ }^{17}$.

Estudos indicam que $15 \%$ dos casos são graves, gerando a necessidade de oxigenioterapia e $5 \%$ de VMI ${ }^{18}$. Outro estudo retratou números superiores, destacando que de 55 pacientes com COVID-19, 67\% desenvolveu SDRA, 63,5\% receberam cânula nasal de alto fluxo (CNAF), 56\% demandaram VMI e 42\% de VNI ${ }^{19}$.

Por ser uma síndrome heterogênea, de alta incidência e morbimortalidade hospitalar, a SDRA é um dos maiores desafios para os profissionais no âmbito da UTI neste perfil de pacientes $^{20}$, sendo o conhecimento destes desafios e métodos eficazes de suporte respiratório essenciais para o manejo, prevenção de infecções e redução das taxas de letalidade ${ }^{17}$.

Um dos desafios é a contaminação por procedimentos geradores de aerossóis: intubação, extubação, broncoscopia, uso de oxigênio nasal de alto fluxo, nebulização, VNI, traqueostomia e ressuscitação cardiopulmonar antes da intubação ${ }^{18,22}$. As respectivas recomendações relacionadas a este desafio estão descritas no Quadro 2.

Quadro 2: Desafios e recomendações para procedimentos geradores de aerossóis

\begin{tabular}{|c|c|}
\hline Desafios & Recomendações \\
\hline $\begin{array}{l}\text { Transmissão da doença por } \\
\text { procedimentos geradores de aerossóis }\end{array}$ & $\begin{array}{l}\text { - Os profissionais devem utilizar os EPIS's aéreos } \\
\text { adequados e etiqueta da tosse e instruir o mesmo } \\
\text { aos pacientes. } \\
\text { - Sugere-se o uso da CNAF ao invés de } \\
\text { oxigenoterapia convencional e VNI. } \\
\text { - As salas de pressão negativa são mais } \\
\text { apropriadas para estes procedimentos. Se não } \\
\text { disponível deve-se realizar em sala única com a } \\
\text { porta fechada e pequena quantidade de } \\
\text { profissionais. } \\
\text { - Em pacientes não-intubados a nebulização não é } \\
\text { preconizada, pois é capaz de originar aerossóis. }\end{array}$ \\
\hline
\end{tabular}




\begin{tabular}{|l|l|}
\hline & Caso necessário deve ser realizada com \\
& inaladores ou espaçadores de medida. \\
& - É indicado circuito de ventilação e aspiração \\
fechado. & \\
& - A broncoscopia deve ser extremamente evitada. \\
\hline Traqueostomia & - Apesar de também produzir aerossóis, para \\
& $\begin{array}{l}\text { contribuir com as intervenções de enfermagem e } \\
\text { favorecer o desmame é recomendada com as } \\
\text { devidas medidas de proteção. }\end{array}$ \\
\hline Intubação & - Deve ser realizada pelo profissional mais bem \\
preparado e protegido.
\end{tabular}

Outro desafio é efetividade e empregabilidade de técnicas respiratórias como CNAF, VNI, ECMO, pressão positiva expiratória final (PEEP), recrutamento alveolar e posição prona em pacientes com COVID-19. O CNAF é uma terapia utilizada para tratamento da hipóxia em pacientes com a COVID-19. Ao comparar o uso da CNAF com a oxigenioterapia convencional e VNI, estudiosos constataram que o uso da CNAF está associado a menores ocorrências de intubação, isto se torna um achado relevante na atual situação enfrentada, visto que com menos intubações haverá mais leitos de UTI e ventiladores disponíveis ${ }^{22}$.

Além disso, estudos indicam que o CNAF pode ser mais confortável e oferecer menos chances de contaminação que a VNI e condições semelhantes de contágio que oxigenioterapia convencional o que anteriormente era um temor dos profissionais ${ }^{22}$. Sendo assim, apesar de alguns autores indicarem evitar o uso de CNAF em pacientes com COVID-19 em decorrência do medo transmissão, a utilização em uma sala de pressão negativa ${ }^{18,22}$, devidamente monitorada, em um ambiente em que a intubação é facilitada e a equipe estejam usando os devidos EPIs é altamente recomendada ${ }^{22}$.

Não existem evidências que amparam a utilização de VNI em pacientes com COVID19, acredita-se que a mesma pode estar relacionada a maior ocorrência de infecção entre os profissionais por gerar aerossóis, altos índices de falha, necessidade de intubação e mortalidade ${ }^{22}$. A ECMO apresenta efeitos positivos no caso da SDRA como reduzir a mortalidade, porém precisa de muito recursos, deste modo o emprego nos perfis mais graves é preconizado ${ }^{18,21}$.

Quanto ao valor de PEEP usado nos pacientes em VM, afim de melhorar a oxigenação, evitar atelectasias e consequentes lesões pulmonares induzidas pelo respirador, indica-se que um número mais alto de PEEP pode ser capaz de reduzir a mortalidade em pacientes, desde que 
haja monitoramento ${ }^{22}$. A posição prona promove uma ventilação mais homogênea e melhora a perfusão e diminui a mortalidade ${ }^{22}$.

A eficácia das técnicas e melhores métodos de aplicação são analisadas no Quadro 3, as quais existem recomendações específicas de acordo com cada caso.

Quadro 3: Desafios e relacionados a técnicas respiratórias utilizadas na SDRA na COVID-19

\begin{tabular}{|c|c|}
\hline Desafios & Recomendações \\
\hline Utilização da CNAF & $\begin{array}{l}\text { - O uso da CNAF está associado a menor } \\
\text { ocorrência de intubação e contaminação. }\end{array}$ \\
\hline Utilização da VNI & $\begin{array}{l}\text { - Pelos altos índices de falha, a utilização rotineira } \\
\text { não é recomendada. } \\
\text { - Pode ser indicada apenas para os casos de SDRA } \\
\text { Leve. } \\
\text { - Os profissionais devem utilizar os EPISs } \\
\text { rigorosos em caso de uso pós-extubação e em } \\
\text { pacientes com Doença Pulmonar Obstrutiva } \\
\text { Crônica. }\end{array}$ \\
\hline Utilização da ECMO & $\begin{array}{l}\text { - Usar ECMO é recomendado nos serviços } \\
\text { disponíveis. } \\
\text { - Na ausência da ECMO no hospital, os pacientes } \\
\text { mais graves devem ser encaminhados a centros de } \\
\text { ECMO. }\end{array}$ \\
\hline Utilização da PEEP & $\begin{array}{l}\text { - Sugere-se utilização de PEEP mais alta em } \\
\text { SDRA moderada e grave. Contudo, os médicos } \\
\text { devem monitorar constantemente a ocorrência de } \\
\text { barotrauma. }\end{array}$ \\
\hline Manobras de recrutamento & $\begin{array}{l}\text { - Podem ser consideradas em pacientes com } \\
\text { SDRA, avaliando caso a caso. }\end{array}$ \\
\hline Posição prona & $\begin{array}{l}\text { - É uma técnica eficaz nos casos de SDRA } \\
\text { reduzindo a mortalidade. } \\
\text { - Em casos graves de SDRA é recomendada por } \\
12 \text { a } 16 \text { horas por dia. }\end{array}$ \\
\hline
\end{tabular}




\begin{tabular}{|l|l|}
\hline & $\begin{array}{l}\text { - A equipe deve ser treinada para que a técnica } \\
\text { seja executada em segurança e sem complicações. }\end{array}$ \\
\hline
\end{tabular}

\section{Terapia Medicamentosa reaproveitadas, experimentais e adjuvantes}

Apesar de não haver terapias específicas aprovadas no Brasil para a SDRA ao Coronavírus 2, vários agentes têm sido usados em ensaios clínicos. Possíveis opções de tratamento, incluindo agentes imunossupressores, antivirais, imunomoduladores e terapias adjuvantes serão abordadas com o intuito de gerar recomendações no manejo da COVID-19. (Quadros 4 e 5).

\section{Terapias reaproveitadas e experimentais}

Cloroquina - É utilizada no tratamento da Malária e doenças reumáticas devido aos seus efeitos imunomoduladores. A cloroquina possui atividade in vitro contra o coronavírus 2 com benefício potencial em inibir a exacerbação da pneumonia causada pela infecção em relatos de uso na dose oral de $500 \mathrm{mg}$ a cada 12 ou $24 \mathrm{~h}$ com duração variável de 5 a 10 dias $^{25,25}$. Contudo, seu uso contra a COVID-19 deve ser circunscrito a ensaios clínicos randomizados com acompanhamento clínico rigoroso, uma vez que sua administração apresenta alto de risco de reações adversas graves e interações medicamentosas importantes no paciente crítico, além de possuir uma estreita margem de segurança - não administrar doses iguais ou acima de 30 $\mathrm{mg} / \mathrm{kg}^{2}$.

Hidroxicloroquina - Mostra-se mais potente, quando comparada com a cloroquina, em um estudo in vitro. Apesar de possuir o mesmo mecanismo de ação, dados preliminares mostraram que a proporção de pacientes que apresentaram resultados negativos de PCR diferiu significativamente entre os grupos testados; 6 dias após o início do tratamento com Hidroxicloroquina, $70 \%$ dos pacientes estavam curados em comparação com $12,5 \%$ no grupo controle. Nesse estudo, a Hidroxicloroquina foi administrada em uma posologia alternativa de $200 \mathrm{mg}$ 8/8h por 10 dias $^{3}$. Existem, outras duas posologias possíveis, a depender das especificidades de cada paciente - $400 \mathrm{mg}$ 12/12h por um dia seguido de $200 \mathrm{mg}$ 12/12h por 4 dias ou $400 \mathrm{mg} /$ dia por 5 dias. Os riscos associados são os mesmos apresentados pela Cloroquina, devendo o seu uso permanecer restrito aos pacientes internados ${ }^{25}$.

Lopinavir/Ritonavir - Dois inibidores de proteases usados no tratamento da SDRA também se encontram no rol de medicamentos promissores contra a COVID-19, usados com regime de administração de $400 \mathrm{mg} / 100 \mathrm{mg}, 12 / 12 \mathrm{~h}$ por 14 dias $^{25}$. Estudos in vitro e em modelos animais mostram atividade contra outros tipos de coronavírus, como o MERS-CoV ${ }^{24}$. Porém, um ensaio 
clínico randomizado realizado com 199 pacientes não mostrou diferença significativa nem no tempo de melhora, nem na taxa de mortalidade, além de estar associado a mais efeitos adversos 27. Apesar desses primeiros dados divulgados, o tratamento para a COVID-19 com Lopinavir e Ritonavir ainda é uma das alternativas da OMS, que aguarda os resultados dos estudos em andamento. Então, o uso dessa combinação não é recomendado devido à falta de ensaios clínicos que corroborem os resultados obtidos em modelos animais.

Oseltamivir - O Oseltamivir é um inibidor de neuroaminidase aprovado para o tratamento da gripe e não possui atividade in vitro documentada contra a SARS-CoV-2. Como o surto de COVID-19 na China ocorreu inicialmente durante o pico da sazonalidade da gripe, uma grande proporção de pacientes recebeu terapia empírica com Oseltamivir até a descoberta da SARSCoV-2 como causa da COVID-1928. Vários ensaios clínicos atuais incluem o seu uso, porém, não como uma intervenção e sim como grupo de comparação. Segundo o Ministério da Saúde, é recomendável o uso de Oseltamivir em pacientes com Síndrome Respiratória Aguda Grave sem diagnóstico etiológico, conforme protocolo atual de SRAG. Contudo, a indicação deverá ser revista após o exame etiológico, uma vez que o Oseltamivir não possui atividade contra SARS-CoV-2 29 .

Remdesivir (GS-5734) - Trata-se de um pró-fármaco análogo de adenosina em investigação. Seu primeiro uso clínico foi no tratamento do Ebola e atualmente é considerado o medicamento mais promissor com relação a priorização de pesquisa de agentes terapêuticos ${ }^{30}$. Além de demonstrar inibição eficaz de SARS-CoV-2 em estudos in vitro, o Remdesivir mostrou-se mais eficaz quando comparado ao grupo controle em animais sendo inclusive superior ao tratamento com Lopinavir/Ritonavir ${ }^{31,32,33}$. A segurança e farmacocinética do Remdesivir foram avaliadas em ensaios clínicos de fase 1 . Infusões intravenosas de 3 e $225 \mathrm{mg}$ foram bem toleradas sem qualquer evidência de toxicidade hepática ou renal. A dose atual sob investigação segue o regime terapêutico de dose única de $200 \mathrm{mg}$ seguida de infusão diária de $100 \mathrm{mg}^{34}$. Estudos clínicos com o intuito de avaliar a segurança e atividade antiviral do Remdesivir em pacientes com COVID-19 leve a moderado ou grave estão em andamento. De acordo com os ensaios já publicados, a inclusão deste agente para o tratamento da COVID-19 pode ser considerada. Vale ressaltar que, por não ser um medicamento registrado pelo órgão sanitário responsável, o Remdesivir deve ser obtido por uso compassivo, acesso expandido ou inscrição em um ensaio clínico $^{25}$.

Quadro 4: Desafios e recomendações no tratamento da COVID-19. 


\begin{tabular}{|c|c|c|}
\hline Terapia & Desafios & Recomendações \\
\hline Cloroquina & $\begin{array}{l}\text { Reações adversas graves tais } \\
\text { como prolongamento do } \\
\text { intervalo QT, hemólise, } \\
\text { hipoglicemia, retinopatia. }\end{array}$ & $\begin{array}{l}\text { - Uso circunscrito a ensaios } \\
\text { clínicos randomizados } \text { com } \\
\text { acompanhamento } \\
\text { rigoroso. }\end{array}$ \\
\hline Hidroxicloroquina & $\begin{array}{l}\text { Apesar de mostrar-se mais } \\
\text { potente quando comparada com a } \\
\text { cloroquina, apresenta os mesmos } \\
\text { riscos associados. }\end{array}$ & $\begin{array}{l}\text { - Uso circunscrito a ensaios } \\
\text { clínicos randomizados } \\
\text { acompanhamento } \\
\text { rigoroso. }\end{array}$ \\
\hline Lopinavir/Ritonavir & $\begin{array}{l}\text { Ensaios clínicos limitados - } \\
\text { associado a mais efeitos } \\
\text { adversos. }\end{array}$ & $\begin{array}{l}\text { - Uso não recomendado na } \\
\text { COVID-19 até a publicação de } \\
\text { mais ensaios clínicos. }\end{array}$ \\
\hline Oseltamivir & $\begin{array}{l}\text { Não possui atividade contra } \\
\text { SARS-CoV-2. }\end{array}$ & $\begin{array}{l}\text { - Não administrar uma vez } \\
\text { confirmada COVID-19. }\end{array}$ \\
\hline Remdesivir & $\begin{array}{l}\text { Medicamento não registrado. } \\
\text { Uso compassivo, acesso } \\
\text { expandido ou inscrição em } \\
\text { ensaio clínico. }\end{array}$ & $\begin{array}{l}\text { - A inclusão deste agente para o } \\
\text { tratamento da COVID-19 pode } \\
\text { ser considerada. }\end{array}$ \\
\hline
\end{tabular}

\section{Terapias adjuvantes}

Corticosteroides - A hiperativação imunológica que ocorre em pacientes com coronavírus levando a produção excessiva de citocinas pró-inflamatórias tem sido citada como justificativa para o uso de corticosteroides no tratamento da COVID-19. Contudo, os ensaios clínicos que abordam esta questão são limitados, trazendo resultados contraditórios ${ }^{25}$. Um relatório publicado na China relata que o uso de Metilprednisolona na dose de $1-2 \mathrm{mg} / \mathrm{kg} / \mathrm{dia}$ por 5 a 7 dias foi associado a uma duração menor de uso suplementar de oxigênio e melhores resultados radiográficos ${ }^{35}$. Ainda na mesma linha, uma revisão sistemática realizada com pacientes hospitalizados com pneumonia adquirida na comunidade mostrou que o uso de corticosteroides pode reduzir a necessidade de ventilação mecânica, diminuindo, assim, o tempo de hospitalização ${ }^{36}$. No entanto, vale ressaltar que as terapias adotadas não eram homogêneas, o que dificulta uma generalização para o contexto atual. Por outro lado, uma revisão recente de 15 estudos de coorte sobre influenza e sobre coronavírus mostrou uma associação entre o uso de corticosteroides e o aumento da mortalidade. Da mesma maneira, esses estudos são limitados 
por apresentarem uma heterogeneidade significativa ${ }^{37}$. Assim, devido a inconsistência dos dados, o uso rotineiro de corticosteroides sistêmicos para tratamento de pneumonia viral ou SRAG não é aconselhado ${ }^{29}$.

Antibacterianos - O uso de antibacterianos em pacientes com COVID-19 baseia-se na extrapolação de dados de outras pneumonias virais, particularmente influenza. Contudo, por apresentarem sintomas semelhantes aos de uma infecção viral, a identificação de uma coinfecção bacteriana é desafiadora ${ }^{37}$. Os pacientes co-infectados por bactérias apresentam maior probabilidade de apresentar choque (21\% versus $10 \%)$, necessitar de ventilação mecânica no momento da admissão na UTI (63\% versus 52\%) e ter maior tempo de permanência na UTI (7 versus 6 dias). Por esta razão, em pacientes com COVID-19 e insuficiência respiratória, é recomendável o tratamento antimicrobiano empírico ${ }^{38}$.

Tocilizumab - O uso de anticorpos monoclonais direcionados contra citocinas inflamatórias encontra-se justificado pela "tempestade de citocinas", termo utilizado para definir uma resposta imune amplificada ${ }^{39}$. A interleucina-6 (IL-6), alvo farmacológico do Tocilizumab, parece ter papel importante nessa inflamação desregulada ${ }^{40}$. Estudos com pacientes graves parecem demonstrar sucesso. Um relatório de 21 pacientes com COVID-19 mostrou que o uso de Tocilizumab $400 \mathrm{mg}$ foi associado à melhora clínica em $91 \%$ dos casos. Contudo, a ausência de um grupo controle limita a interpretação do efeito específico do medicamento, razão pela qual seu uso na prática clínica deve ser realizado com cautela até que mais estudos sejam concluídos ${ }^{41,42}$.

Quadro 5: Desafios e recomendações na terapia adjuvante da COVID-19.

\begin{tabular}{|l|l|l|}
\hline \multicolumn{1}{|c|}{ Terapia } & \multicolumn{1}{|c|}{ Desafios } & \multicolumn{1}{|c|}{ Recomendações } \\
\hline Corticosteroides & $\begin{array}{l}\text { Ensaios clínicos limitados e } \\
\text { resultados contraditórios. }\end{array}$ & $\begin{array}{l}\text { Não administrar rotineiramente } \\
\text { corticosteroides sistêmicos para } \\
\text { tratamento de pneumonia viral ou } \\
\text { SRAG fora dos ensaios clínicos, a } \\
\text { menos que sejam indicados por outro } \\
\text { motivo. }\end{array}$ \\
\hline Antibacterianos & $\begin{array}{l}\text { Obtenção de amostras de } \\
\text { qualidade para escalonamento/ } \\
\text { descalonamento. }\end{array}$ & $\begin{array}{l}\text { - Uso empírico - administração dentro } \\
\text { de uma hora da avaliação inicial de } \\
\text { pacientes com sepse - seguido de }\end{array}$ \\
\hline
\end{tabular}




\begin{tabular}{|l|l|l|}
\hline & & $\begin{array}{l}\text { terapia guiada por exames } \\
\text { laboratoriais. }\end{array}$ \\
\hline Tocilizumab & Ensaios clínicos limitados. & $\begin{array}{l}\text { - Não há recomendações sobre o seu } \\
\text { uso. Ensaios clínicos em curso. }\end{array}$ \\
\hline
\end{tabular}

\section{Prevenção de Infecções}

Os profissionais de saúde atuantes na linha de frente estão expostos a uma série de riscos de contaminação pelo SARS-CoV-2. Isso ocorre porque a maioria dos procedimentos de manejo do paciente envolvem manipulação de tecidos, fluidos corpóreos e aerossóis ${ }^{40}$. O risco de adoecimento do profissional implica na redução do corpo clínico hospitalar, o que pode reduzir a capacidade de cuidado dos doentes. Além disso, uma vez contaminado, ele também poderá disseminar partículas virais, aumentando a susceptibilidade a infecções hospitalares ${ }^{41}$.

Estudo recente realizado com 138 pacientes com COVID-19, evidenciou que destes, $41,3 \%$ foram infectados no hospital, sendo $28 \%$ profissionais de saúde e $12,3 \%$ pacientes já hospitalizados por outras comorbidades ${ }^{42}$. É possível que existam diferentes rotas de transmissão do novo coronavírus, como a transmissão direta (inalação de gotículas por tosse, espirro), contato via mucosas bucal, nasal e ocular e transmissão indireta, através de superfícies contaminadas ${ }^{43,44}$.

Portanto, o controle e prevenção de infecção, em ambiente hospitalar é imperativo para a contingência da doença. Para isso, é necessário que sejam adotadas medidas de precaução para gotículas e aerossóis em determinados procedimentos, bem como o uso de EPIs adequados. A descontaminação de superfícies com agentes capazes de erradicar vírus presentes em superfícies, adequação das visitas aos pacientes, de modo a minimizar a exposição destes e o transporte seguro do paciente, quando necessário, também são fatores relacionados ao sucesso do controle da infecção. O controle de visitas é outro fator para controle de infecções. Vamos discutir cada medida e o Quadro 6 trará a síntese.

\section{Equipamento de Proteção Individual}

Os EPIs recomendados para a assistência a pacientes com suspeita ou confirmação da COVID-19 incluem, em ordem de uso: gorro para proteção capilar, máscara N95, óculos de proteção ou face shields, luvas com punho longo e macacão resistente a líquidos, que deve ficar sobre a roupa privativa, capote e sapatos impermeáveis. Além disso, o profissional deve realizar 
antissepsia de mãos com preparação alcoólica $70 \%$ ou água e sabão neutro, antes da paramentação, realizada na antecâmara e após a desparamentação, tanto na remoção de luvas e capotes, ainda no ambiente em que o paciente se encontra, quanto ao término da remoção dos EPIs, na antecâmara ${ }^{45,46}$.

A Agência Nacional de Vigilância Sanitária (ANVISA) segmentou o uso de EPI de acordo com a assistência prestada e proximidade com pacientes suspeitos e contaminados. Assim, apenas em procedimentos geradores de aerossóis, tais como intubação e aspiração orotraqueal, ressuscitação cardiopulmonar, e broncoscopias, é necessário o uso de gorro e máscara N95 ou PFF2. Pacientes com sinais de infecção respiratória, tais como tosse e espirro, também deverão utilizar máscara cirúrgica ${ }^{47}$.

Além do cuidado durante o atendimento, é importante que a equipe hospitalar se atenha a desinfecção das superfícies. Conoravírus são capazes de sobreviver em superfícies de plástico, tecidos - inclusive EPI, madeira, vidro e azulejos, por aproximadamente 72 horas $^{48,50}$. Entretanto, uma recente revisão da literatura, evidenciou que o vírus pode permanecer infeccioso por até 9 dias em superfícies e objetos inanimados. Apesar disso, o uso de hipoclorito de sódio $0,1 \%$ ou etanol nas concentrações entre $62 \%$ a $71 \%$, reduziram significativamente a carga viral, após 1 minutos de exposição ${ }^{51}$.

Além desses, soluções de quaternário de amônio e iodóforos, demonstraram eficácia na inativação viral. Portanto, é recomendado que superfícies possivelmente contaminadas, devam ser limpas com detergentes neutros, e, em seguida, realizada a desinfecção com esses agentes a cada 6 horas. Caso existam sujidades, estas devem ser removidas com tecido ou papel absorvente, antes da limpeza ${ }^{47}$.

\section{Transporte do paciente intra-hospitalar e o Controle de Visitas}

O transporte adequado envolve o uso de EPIs limpos, com uso de máscara N95, e apenas um, será encarregado de tocar superfícies. Para o paciente em VM, é necessário verificar a necessidade de aspiração do tubo orotraqueal (TOT), o cuff e a fixação do TOT, ajustar e reforçar conexões dos circuitos. Deve-se conferir as bombas de infusão, ajustar $\mathrm{FiO}_{2}$ para $100 \%$ e conectar o VM ao cilindro de oxigênio diretamente na válvula do próprio cilindro ${ }^{52}$. Após o transporte, o profissional deverá seguir todos os procedimentos para a remoção dos EPIs ${ }^{53}$.

Para pacientes adultos internados com suspeita ou confirmação de COVID-19, visitas não são recomendadas. A equipe poderá viabilizar a visita virtual. Demais pacientes da UTI 
adulto, poderão receber apenas um visitante ao dia, em horário estipulado. Pessoas que manifestem síndromes gripais não poderão realizar visitas e as do grupo de risco devem evitar $^{53,54}$.

Quadro 6. Desafios e recomendações para prevenção de infecções

\begin{tabular}{|c|c|}
\hline Desafios & Recomendações \\
\hline EPIs & $\begin{array}{l}\text { - Realizar higiene de mãos com manipulação alcoólica ou sabão } \\
\text { neutro e água. } \\
\text { - Utilizar máscara N95, além dos demais EPIs, para } \\
\text { procedimentos geradores de aerossóis. } \\
\text { - Utilizar máscara cirúrgica, luvas, óculos ou face shields e } \\
\text { avental, em demais procedimentos. }\end{array}$ \\
\hline $\begin{array}{l}\text { Descontaminação } \\
\text { superfícies }\end{array}$ & $\begin{array}{l}\text { - Realizar desinfecção após a limpeza com sabão neutro. } \\
\text { - Dar preferência a soluções de hipoclorito de sódio } 0,1 \% \text { e } \\
\text { álcool } 70 \% \text {. } \\
\text { - Sempre realizar limpeza e desinfecção da maca e } \\
\text { equipamentos anexados, após o transporte do paciente. } \\
\text { - Realizar desinfecção das superfícies potencialmente } \\
\text { contaminadas, a cada } 6 \text { horas. }\end{array}$ \\
\hline Transporte intra-hospitalar & $\begin{array}{l}\text { - Equipe envolvida deverá utilizar EPIs limpos e permanecer } \\
\text { com a máscara N95. } \\
\text { - Verificar Cuff e necessidade de aspiração orotraqueal prévios } \\
\text { ao transporte. } \\
\text { - Verificar conexão dos circuitos. }\end{array}$ \\
\hline Visitas à UTI & $\begin{array}{l}\text { - Evitar visitantes do grupo de risco. } \\
\text { - Pessoas com síndromes gripais não poderão realizar visitas. } \\
\text { - Evitar grande fluxo de pessoas. } \\
\text { - Avaliar a possibilidade de chamada por vídeo. }\end{array}$ \\
\hline
\end{tabular}

\section{Gestão da Mortalidade}

Quando o desfecho do paciente grave por COVID-19 é o falecimento, os profissionais de saúde da UTI deverão lidar com o desafio do risco de contaminação ${ }^{1,2}$. Além disso, deverão estar seguros sobre os trâmites para encaminhar o corpo para a necropsia, emissão da declaração 
de óbito (DO) específico da doença, comunicar e orientar os familiares e amigos sobre o óbito e regras do velório e funerais ${ }^{56}$. Cada um dos aspectos será apresentado e discutido e os desafios e recomendações estão no Quadro 7.

O manejo do corpo falecido deverá ocorrer por quantidade de profissionais necessária, os quais precisam utilizar todos os EPIs recomendados e já descritos. Ao final do manejo do corpo, todo o material e rouparia deverão ser descartados em local adequado ${ }^{56}$. Os profissionais deverão manipular o corpo o mínimo possível e evitar procedimentos que gerem gases ou extravasamento de fluidos corpóreos. Quando possível, a embalagem do corpo deve seguir três etapas: enrolar o corpo com lençóis; colocar o corpo em um saco impermeável; colocar um segundo saco e desinfetar com álcool $70 \%$, solução clorada $0,5 \%$ a $1 \%$ ou outro saneante regularizado pela ANVISA. Além da identificação rotineira do corpo, deve-se acrescentar a terminologia: "COVID 19, agente biológico classe de risco 3." "56 . A maca de transporte deve ser utilizada apenas para esse fim. Porém, se for necessário reutilizar, deve-se realizar a desinfecção com álcool a 70\%, solução clorada 0,5 a $1 \%$ ou outro saneante autorizado pela ANVISA. É necessário, ainda, registrar o nome, data e a atividade dos profissionais que realizaram os cuidados pós morte, incluindo o pessoal da limpeza do leito/quarto ${ }^{56}$.

Quanto ao preenchimento da DO, na $10^{\mathrm{a}}$ Revisão da Classificação Estatística Internacional de Doenças e Problemas Relacionados com a Saúde (CID-10), há o código de emergência U07.1 para o diagnóstico da doença respiratória aguda, devido à COVID-19 e a OMS indica o uso desse código na DO. Contudo, a CID-10 no Brasil não possui esse código. Assim, recomenda-se o código B34.2 (Infecção por Coronavírus de Localização não Especificada). Óbitos ocorrido por doença respiratória aguda à COVID-19, utilizar também U04.9 (Síndrome Respiratória Aguda Grave - SARS) ${ }^{56}$.

No caso do paciente falecido com a doença de coronavírus, em decorrência das restrições de visitas, o profissional deverá decidir sobre o modus operandi da comunicação, se presencialmente, via telefone ou videoconferência. A comunicação do óbito deve considerar os princípios de humanização e ser baseada em protocolos consolidados de transmissão más notícias, para produzir efeitos de cuidado, que minimizam o sofrimento envolvido.

O reconhecimento do corpo deve ocorrer apenas por um familiar/responsável e com distância mínima de 2 metros entre eles. Se houver necessidade de aproximação, o familiar deverá fazer uso de máscara cirúrgica, luvas e aventais de proteção. Se o ambiente for muito 
limitado para o reconhecimento, pode ser por meio de fotografias, evitando contato ou exposição ${ }^{56}$.

Os familiares deverão ser orientados de que os velórios e funerais não são recomendados. Também devem ser informados de que o corpo será entregue em uma urna funerária fechada e que assim deve permanecer. No caso de velório, a família precisa ser instruída a seguir condutas que evitem transmissão do vírus, a saber: o local deve ser aberto ou ventilado ${ }^{56}$; não se deve ultrapassar 10 pessoas; pessoas do grupo de risco ou com sinais e sintomas da doença não devem comparecer ${ }^{56}$. Com o avanço da pandemia, cada vez mais fica contraindicada a realização de velório.

Quadro 7. Desafios e recomendações na gestão da mortalidade por COVID-19

\begin{tabular}{|c|c|}
\hline Desafios & Recomendações \\
\hline Manejo do corpo falecido & $\begin{array}{l}\text { - Quantidade de profissional não excedente. } \\
\text { - Utilizar EPIs recomendados. } \\
\text { - Descartar o material e roupas em local } \\
\text { apropriado. } \\
\text { - Se possível, embalar o corpo em } 3 \text { etapas: } \\
\text { lençóis, saco impermeável interno e externo. } \\
\text { - Identificar a embalagem com os dados do } \\
\text { falecido, incluindo: "COVID-19, agente } \\
\text { biológico classe de risco } 3 \text { " e realizar } \\
\text { desinfecção. } \\
\text { - Desinfectar a maca. } \\
\text { - Registrar nome, data e a atividade dos } \\
\text { profissionais que realizaram os cuidados pós } \\
\text { morte, incluindo o pessoal da limpeza do } \\
\text { leito/quarto. }\end{array}$ \\
\hline Declaração de óbito & $\begin{array}{l}\text { - No Brasil, utilizar o código B34.2 (Infecção } \\
\text { por Coronavírus de Localização não } \\
\text { Especificada) e óbitos ocorrido por doença } \\
\text { respiratória aguda à COVID } 19 \text { e acrescentar } \\
\text { U04.9 (Síndrome Respiratória Aguda Grave } \\
\text { - SARS). }\end{array}$ \\
\hline
\end{tabular}




\begin{tabular}{|l|l|}
\hline Orientações aos familiares e amigos & - Comunicação do óbito adaptada, \\
humanizada e com uso de protocolos de \\
transmissão de más notícias. \\
- O reconhecimento do corpo limitado a uma \\
pessoa, com distância mínima de 2 metros. \\
Se for imperativa a aproximação, familiar \\
deve usar EPI. \\
- Por critério de segurança, é possível \\
reconhecimento do corpo por meio de \\
fotografia. \\
- Equipe de UTI deve fornecer as \\
informações específicas sobre as condições \\
do velório para evitar transmissão do vírus.
\end{tabular}

Fonte: Ministério da Saúde ${ }^{56}$.

\section{Gerenciamento de equipe de UTI}

Com a pandemia, podemos ter a situação do aumento da jornada de trabalho e baixa de profissionais. Por outro lado, altas taxas de carga de trabalho na UTI estão associadas a um aumento na mortalidade dos pacientes. Poderá ser necessário aumento da equipe com profissionais não treinados em terapia intensiva. Todos os profissionais deverão ser treinados sobre gerenciamento geral de terapia intensiva e protocolos específicos do COVID-19.

Essas condições de trabalho, além de impactar diretamente na qualidade da assistência, podem produzir efeitos na saúde física e mental dos profissionais de saúde. $\mathrm{O}$ risco de desenvolvimento de problemas de saúde mental fica aumentado, em função do medo e da ansiedade para lidar com uma doença nova, ainda pouco esclarecida e altamente contagiosa ${ }^{8} \cdot \mathrm{A}$ oferta de treinamento da equipe de UTI também produz efeito de proteger a saúde mental dos profissionais, pois fomenta o sentimento de autoeficácia. Para fins de saúde mental, é preciso garantir que os profissionais tenham acesso a informações de qualidade sobre: o fluxo e protocolos para manejo do paciente com COVID-19; a utilização e obrigatoriedade do uso correto dos EPIs; o controle do número de profissionais na área de isolamento e sobre as ações efetivas de prevenção de infecções. Limitar a carga horária, de acordo com a legislação vigente, respeitando os horários de intervalo, estabelecer rodízios para realização de tarefas estressantes 
e facilitar períodos de alimentação são outras estratégias para favorecer a saúde mental dos profissionais. É recomendado identificar pessoas com risco psicossocial prévio, profissionais em condição de vulnerabilidade (portadores de doenças crônicas, que residem com filhos pequenos ou familiares idosos) e, com isso, oferecer suporte emocional e alternativas para uma possível readequação das funções. Por fim, disponibilizar uma rede de apoio de saúde mental para os profissionais, incluindo psiquiatras e psicólogos, é altamente indicado ${ }^{57}$ Vejamos o Quadro 8.

Quadro 8. Desafios e Recomendações para gerenciamento de equipe de UTI

\begin{tabular}{|c|c|}
\hline Desafios & Recomendações \\
\hline Problemas de saúde mental e risco psicossocial & $\begin{array}{l}\text { - Manutenção do sentimento de } \\
\text { autoeficácia mediante treinamento e } \\
\text { aquisição de conhecimentos sobre a } \\
\text { doença de coronavírus. } \\
\text { - Alternativas para adequação das funções } \\
\text { - Disponibilizar rede de apoio a saúde } \\
\text { mental }\end{array}$ \\
\hline Segurança dos profissionais & $\begin{array}{l}\text { - Oferta de treinamentos de qualidade. } \\
\text { - Trabalho com foco com foco na } \\
\text { prevenção de infecções. }\end{array}$ \\
\hline Sobrecarga de trabalho & $\begin{array}{l}\text { - Garantir número adequado de } \\
\text { profissionais, incluindo recrutamento de } \\
\text { profissionais externos à UTI. } \\
\text { - Respeitar a carga horária diária, } \\
\text { intervalos e períodos de alimentação. } \\
\text { - Estabelecer rodízios de tarefas } \\
\text { estressantes }\end{array}$ \\
\hline
\end{tabular}

\section{CONSIDERAÇÕES FINAIS}

A comunidade de UTI deve se preparar para os desafios associados à pandemia: organizar antecipadamente os fluxos de trabalho; realizar diagnóstico e isolamentos rápidos; gerenciar a prevenção de infecções; manejar a insuficiência respiratória aguda; treinar equipes para empregar protocolos padrão; conhecer o papel das terapias medicamentosas reaproveitadas, experimentais e adjuvantes; aumentar a capacidade de leitos de UTI; fazer a 
gestão da mortalidade e proteger os profissionais de saúde contra a contaminação hospitalar, exaustão física e problemas de saúde mental.

Para o paciente crítico internado em UTI, ainda são necessários estudos sobre: protocolos de triagem para a UTI; agentes terapêuticos reaproveitados ou experimentais; definição de eficácia da VNI e HFNC; risco de transmissão nosocomial em salas compartilhadas de UTI; clareza sobre o comprometimento cardíaco e a disfunção miocárdica; o papel da ECMO (circulação extracorpórea) e as indicações sobre indicações de corticosteroides.

\section{REFERÊNCIAS}

1. Vincent, J.L; Taccone, F.S. Understanding Pathways to Death in Patients with COVID19. The Lancet. Respiratory Medicine.April 06, 2020. p. 2019-2021. Available from:https://www.thelancet.com/journals/lanres/article/PIIS2213-2600(20)30165$\underline{\text { X/fulltext\#articleInformation }}$

2. Grasselli G; Pesenti A; Cecconi M. Critical care utilization for the COVID-19 outbreak in Lombardy, Italy: early experience and forecast during an emergency response. JAMA. New England Journal of Medicine. March 13, 2020. Available from: https://jamanetwork.com/journals/jama/fullarticle/2763188

3. Xiaobo Y; Yuan Y; Jiqian X; Shu H; Xia J; Liu H; et al. Clinical course and outcomes of critically ill patients with SARS-CoV-2 pneumonia in Wuhan, China: a singlecentered, retrospective, observational study. The Lancet. Respiratory Medicine.February 21, 2020.Available from: https://www.thelancet.com/action/showPdf?pii=S22132600\%2820\%2930079-5

4. Aylward, B; Liang, W. WHO-China Joint Mission. Report of the WHO-China Joint Mission on Coronavirus Disease 2019 (COVID-19). The WHO-China Joint Mission on Coronavirus Disease 2019. February 28, 2020. Available from: https:/www.who.int/docs/default-source/coronaviruse/who-china-joint-mission-on-covid-19final-report.pdf

5. Johns Hopkins University. All rights reserved. Coronavirus Resource Center. Site. Acessado em 16 de Abril de 2020.

6. Agência Brasil. Últimas Notícias Covid-19. Brasil. 2020. Acessado em: 16 de Abril de 2020. 
7. Dawei W; Bo H; Chang H; Fangfang Z; Xing L; Jing Z; et al. Clinical characteristics of 138 hospitalized patients with 2019 novel coronavirus-infected pneumonia in Wuhan, China. JAMA. March 17, 2020. p. 1061-1069. Available from: https://jamanetwork.com/journals/jama/fullarticle/2761044

8. Jason P; Li W; Lowell L; Moritoki E; Chae-Man L; Jigeeshu V.D; et al. Intensive care management of coronavirus disease 2019 (COVID-19): challenges and recommendations. The Lancet. Respiratory Medicine. April 6, 2020.p.1-12. Available from: $\quad$ https://www.thelancet.com/journals/lanres/article/PIIS2213-2600(20)301612/fulltext.

9. Nanshan C; Min Z; Xuan D; Jieming Q; Fengyun G; Yang H; et al. Epidemiological and clinical characteristics of 99 cases of 2019 novel coronavirus pneumonia in Wuhan, China: a descriptive study. The Lancet. February 15, 2020. Available from: $\underline{10.1016 / \mathrm{S} 0140-6736(20) 30211-7}$

10. Waleed A; Morten H.M; Yaseen M.A; Mark L; Michelle N.G; Eddy F, Simon O; et al. Surviving Sepsis Campaign: guidelines on the management of critically ill adults with Coronavirus Disease 2019 (COVID-19). Intensive Care Med. March, 2020. Available from:https://www.sccm.org/getattachment/Disaster/SSC-COVID19-Critical-CareGuidelines.pdf.

11. Wenling W; Yanli X; Ruqin G; Roujian L; Kai H; Guizhen W; et al. Detection of SARSCoV-2 in Different Types of Clinical Specimens. American Medical Association.March,2020. Available from: https://jamanetwork.com/journals/jama/fullarticle/2762997.

12. Ministério da Saúde. Boletim COE COVID-19: Doença pelo coronavírus 2019; Abril,2020.

13. Patel R. Report from the American Society for Microbiology COVID-19 International Summit, 23 March 2020: Value of Diagnostic Testing for SARS-CoV-2/COVID-19. American Society for Microbiology; March 26, 2020. p. 1-5. Available from: https://doi.org/10.1128/mBio.00722-20.

14. Ministério da Saúde. Diretrizes para Diagnóstico e Tratamento da COVID-19, Versão 2. Abril, 2020.

15. Colégio Brasileiro de Radiologia e Diagnóstico por Imagem. Recomendações de uso de métodos de imagem para pacientes suspeitos de infecção pelo COVID-19. Versão 2. Abril, 2020. 
16. Fan E; Brodie D; Slutsky AS. Acute Respiratory Distress Syndrome Advances in Diagnosis and Treatment. JAMA. February 20, 2018. p. 698-710. Available from: 10.1001/jama.2017.21907

17. Xin Y; Jinsong M; Guoxin M; Xingshuo H; Peng Y; Lixin X. The timing and intervention strategy of the new respiratory support for coronavirus pneumonia Chinese. Journal of Tuberculosis and Respiratory Diseases. 2020. p. 177-180. Available from: http://rs.yiigle.com/CN112147202003/1184473.htm

18. Thomas P; Baldwin C; Bissett B; Boden I; Gosselink R; Granger CL; et al. Physiotherapy Management for COVID-19 in the Acute Hospital Setting: Recommendations to guide clinical practice. Journal of Physiotherapy. 2020. Available from: https://doi.org/10.1016/j.jphys.2020.03.011.

19. Yang X; Yuan Y; Xu J; Shu H; Xia J; Liu H; et al. Clinical course and outcomes of critically ill patients with SARS-CoV-2 pneumonia in Wuhan, China: a single-centered, retrospective, observational study. The Lancet. Respiratory Medicine. February 24, 2020. Available from: https://doi.org/10.1016/S2213-2600(20)30079-5.

20. Scala R; Heunks L. Highlights in acute respiratory failure. Eur Respir Rev. March 05, 2018. p. 1-4. Available from: [https://doi.org/10.1183/16000617.0008-2018]

21. Phua J; Weng L; Ling L; Egi M; Lim C; Divatia JV; et al. Intensive care management of coronavirus disease 2019 (COVID-19): challenges and recommendations. The Lancet. Respiratory Medicine. April 06, 2020. p. 1-12. Available from: https://doi.org/10.1016/S2213-2600(20)30161-2.

22. Alhazzani W; Moller M.H; Arabi Y.M; Loeb M; Gong MN; Fan E; et al. Surviving Sepsis Campaign: guidelines on the management of critically ill adults with Coronavirus Disease 2019 (COVID-19). Intensive Care Med. 2020 Available from: https://doi.org/10.1007/s00134-020-06022-5.

23. Associação de Medicina Intensiva Brasileira. Manuseio do paciente com infecção pelo Coronavírus COVID-19 e pneumonia e insuficiência respiratória. SP, março 2020.

24. Smith T; Bushek J; LeClaire A; Prosser T. COVID-19 Drug Therapy. Elsevier. 2020. Available from: https://www.elsevier.com/_ data/assets/pdf file/0007/988648/COVID19-Drug-Therapy_Mar-2020.pdf

25. Sanders J.M; Monogue M.L; Jodlowski T.Z; Cutrell J.B. Pharmacologic Treatments for Coronavirus Disease 2019 (COVID-19). JAMA 2020. doi:10.1001/jama.2020.6019. Available from: https://jamanetwork.com/journals/jama/fullarticle/2764727 
26. Yao X; Ye F; Zhang M; Cui C; Huang B; Niu P; et al. In vitro antiviral activity and projection of optimized dosing design of hydroxychloroquine for the treatment of Severe Acute Respiratory Syndrome Coronavirus 2 (SARS-CoV-2). Clin Infect Dis. 2020 [Epub ahead of print] doi: 10.1093/cid/ciaa237. Available from: https://www.ncbi.nlm.nih.gov/pme/articles/PMC7108130/

27. Cao B, Wang Y, Wen D, Liu W, Wang J, Fan G, et al. A trial of lopinavir-ritonavir in adults hospitalized with severe COVID-19. $N$ Engl J Med 2020. Available from: https://www.nejm.org/doi/full/10.1056/NEJMoa2001282

28. Jianfeng Xie; Zhaohui Tong; Xiangdong Guan; Bin Du; Haibo Qiu; Arthur S. Slutsky. Critical care crisis and some recommendations during the COVID-19 epidemic in China. Intensive Care Med. March; 2020. Available from: https://doi.org/10.1007/s00134-02005979-7.

29. Ministério da Saúde. Protocolo de manejo clínico da COVID-19 na Atenção Especializada [recurso eletrônico] - 1. ed. rev. 2020.

30. World Health Organization. Informal consultation on prioritization of candidate therapeutic agents for use in novel coronavirus 2019 infection. 2020.

31. Wang M; Cao R; Zhang L; Yang X; Liu J; Xu M; et al. Remdesivir and chloroquine effectively inhibit the recently emerged novel coronavirus (2019-nCoV) in vitro. Cell Res. 2020; 30:269-271. Available from: https://www.nature.com/articles/s41422-020$\underline{0282-0}$

32. Sheahan TP; Sims AC; Leist SR; Schafer A; Won J; Brown AJ; et al. Comparative therapeutic efficacy of remdesivir and combination lopinavir, ritonavir, and interferon beta against MERS-CoV. Nat Commun. 2020; 11:222. Available from: https://www.nature.com/articles/s41467-019-13940-6

33. De Wit E; Feldmann F; Cronin J; Jordan R; Okumura A; Thomas T; et al. Prophylactic and therapeutic remdesivir (GS-5734) treatment in the rhesus macaque model of MERSCoV infection. Proc Nat Acad Sci USA. 2020; 117(12):6771-6776. Available from: https://www.ncbi.nlm.nih.gov/pmc/articles/PMC7104368/

34. World Health Organization. WHOR\&D blueprint: ad-hoc expert consultation on clinical trials for Ebola therapeutics. 2018.

35. Wang Y; Jiang W; He Q; Wang C; Wang B; Zhou P; et al. Early, lo-dose and short-term application of corticosteroid treatment in patients with severe COVID-19 pneumonia: single-center experience from Wuhan, China. medRxiv 2020. Available from: https://www.medrxiv.org/content/10.1101/2020.03.06.20032342v1 
36. Siemieniuk R.A; Meade M.O; Alonso C.P; Briel M; Evaniew N; Prasad M; et al. Corticosteroid therapy for patients hospitalized with community-acquired pneumonia: a systematic review and meta-analysis. Ann Intern Med 2015; 163:519-528. Available from: http://unmhospitalist.pbworks.com/w/file/fetch/99125931/0000605-20151006000397.pdf

37. Alhazzani W; Moller M.H; Arabi Y.M; Loeb M; Gong M.N; Fan E; et al. Surviving Sepsis Campaign: guidelines on the management of critically ill adults with Coronavirus Disease 2019 (COVID-19). Intensive Care Med. 2020. Available from: https://www.ncbi.nlm.nih.gov/pmc/articles/PMC7101866/

38. Rice T.W; Rubinson L; Uyeki T.M; Vaughn F.L; John B.B; Miller R.R; et al. Critical illness from 2009 pandemic influenza A virus and bacterial coinfection in the United States. Crit Care Med 2012; 40:1487-1498. Available from: https://www.ncbi.nlm.nih.gov/pmc/articles/PMC3653183/

39. Guan W.J; Ni Z.Y; Hu Y; Liang WH; Ou CQ; He J.X; et al. Clinical characteristics of Coronavirus Disease 2019 in China. $N$ Engl J Med 2020. Available from: https://www.ncbi.nlm.nih.gov/pmc/articles/PMC7092819/

40. Mehta P; McAuley D.F; Brown M; Sanchez E; Tattersall RS; Manson JJ. COVID-19: consider cytokine storm syndromes and immunosuppression. Lancet 2020; 020;395(10229):1033-1034. Available from:

https://www.thelancet.com/pdfs/journals/lancet/PIIS0140-6736(20)30628-0.pdf

41. Chan J.W; Yuan S; Kok K.H; Kelvin K.W; Hin C; Yang J; et al. A familial cluster of pneumonia associated with the 2019 novel coronavirus indicating person-to-person transmission: a study of a family cluster. The Lancet. 2020; 395:514-523. Available from: https://www.thelancet.com/journals/lancet/article/PIIS0140-6736(20)30154-9/fulltext

42. Chang D.X.H; Rebaza A; Sharma L.C.S.D. Protecting health-care workers from subclinical coronavirus infection. The Lancet. 2020;8:13. Available from: https://www.thelancet.com/journals/lanres/article/PIIS2213-2600(20)30066-7/fulltext

43. Fei Zhou; Ting Yu; Ronghui Du; Guohui Fan; Ying Liu, Zhibo Liu; et al. Clinical course and risk factors for mortality of adult in patients with COVID-19 in Wuhan, China: a retrospective cohort study. The Lancet. March, 2020. Available from: https://doi.org/10.1016/S0140-6736(20)30566-3.

44. Peng W; Xu X; Li Y; Cheng L; Zhou X. Transmission routes of 2019-nCoV and controls in dental practice. Int Jour of Oral Sci. 2020; 12:9. Available from: https://www.ncbi.nlm.nih.gov/pmc/articles/PMC7054527/ 
45. Wax R.S; Christian M.D. Practical recommendations for critical care and anesthesiology teams caring for novel coronavirus (2019-nCoV) patients. Canadian Jour of Anest. 2020;5:568-576. Available from: https://www.ncbi.nlm.nih.gov/pubmed/32052373

46. Government of Canada . Infection prevention and control for novel coronavirus (2019nCoV): interim guidance for acute healthcare settings. 2020.

47. Liang T. Hanbook of COVID-19 Prevention and Treatment. Zhejang University School of $\quad 2020.2$ Available from: https://www.ephi.gov.et/images/novel coronavirus/Handbook-of-COVID-19-Prevention-andTreatment.pdf

48. ANVISA. Nota técnica GVIMS/GGTES/ANVISA No4/2020. Orientações para serviços de saúde: medidas de prevenção e controle que devem ser adotadas durante a assistência aos casos suspeitos ou confirmados de infecção pelo novo coronavírus (SARS-CoV-2). 2020.

49. Otter J.A; Donsckey C; Douthwaite S; Goldenberg S.D; Weber D.J. Transmission of SARS and MERS coronaviruses and influenza virus in healthcare settings: the possible role of dry surface contamination. Jour of Hosp Infec. 2016;3:235-250. Available from: https://www.ncbi.nlm.nih.gov/pubmed/26597631/

50. Sizun J; Yu M.W; Talbot P.J. Survival of human coronaviruses 229E and OC43 in suspension and after drying on surfaces: a possible source of hospital-acquired infections. $J$ Hosp Infect. 2000; 1:55-60. Available from: https://www.ncbi.nlm.nih.gov/pubmed/11023724

51. Casanova L; Rutala W.A; Weber D.J; Sobsey M.D. Coronavirus survival on healthcare personal protective equipment. Infect Control Hosp Epidemiol.2010. Available from: https://www.cambridge.org/core/journals/infection-control-and-hospitalepidemiology/article/coronavirus-survival-on-healthcare-personal-protectiveequipment/C666673A5460A03D3BB996CDE5019C1B

52. Kampf G; Todt D; Pfaender S; Steinmann E. Persistence of coronaviruses on inanimate surfaces and their inactivation with biocidal agents. Jour of Hosp Infec. 2020. Available from: https:/www.journalofhospitalinfection.com/article/S0195-6701(20)30046-3/fulltext

53. Ebserh. Protocolo: Transporte intra-hospitalar de pacientes em ventilação mecânica com COVID-19. 2020.

54. Sociedade Beneficente Israelita - Albert Einstein. Manejo novo coronavírus (COVID19). 2020. 
55. Ministério da Saúde. Recomendações para acompanhantes elou visitantes nos serviços de atenção especializada em saúde durante pandemia de covid-19. 2020.

56. Ministério da Saúde. Manejo de corpos no contexto do novo coronavírus COVID 19. Brasília/DF. 25 de Março de 2020.

57. Associação de Medicina Intensiva Brasileira-AMIB. Recomendações para o bem-estar emocional da equipe multidisciplinar durante a pandemia pelo Sars-Cov-2: pelo Departamento de Psicologia da AMIB. SP, março 2020. 